\title{
Intrathecal Delivery of Ketorolac Loaded In Situ Gels for Prolonged Analgesic and Anti-Inflammatory Activity in Vertebral Fracture
}

\author{
Shu-E Cao, Sheng-Yang Chen, Jian-Min Tian, Xiao-Ran Zhang, Jun Liu, Hong- \\ Yan Gong and Xiu-Qin Yue* \\ Department of Anesthesiology, The First Affiliated Hospital of Xinxiang Medical University, Weihui, Henan453100, China
}

*For correspondence: Email: yuexiuqin317@gmail.com; Tel/Fax: 0086-0373-4402307

Received: 15 April 2015

Revised accepted: 2 December 2015

\begin{abstract}
Purpose: To develop biodegradable, polymeric in situ gels based on sodium alginate and hydroxypropyl methylcellulose for intrathecal delivery of ketorolac tromethamine (KT) for effective management of pain and inflammation in vertebral fracture.

Method: Ion activated in situ gels were used as implants and were prepared from sodium alginate and hydroxypropyl methylcellulose. The fabricated gels were evaluated for visual appearance, clarity, $\mathrm{pH}$, gelling capacity, drug content, viscosity (using Brookfield viscometer), in vitro drug release (using a fabricated KC cell) and in vivo analgesic and anti-inflammatory activity (by intrathecal administration of in situ gel near the fractured vertebra in a rat model).

Results: The physicochemical properties (visual appearance, clarity, $\mathrm{pH}$, gelling capacity, drug content and viscosity) of in situ gels were acceptable for therapeutic use. KT-loaded gels demonstrated high drug encapsulation efficiency (98.3 - $103.3 \%)$. Further, KT-loaded gels exhibited viscosity in the range of 1.11 to $6 \mathrm{cps}$ at $50 \mathrm{rpm}$ and shear thinning property (rheology testing). Additionally, the gels demonstrated 84.43 to $96.98 \%$ drug release at the end of $12 \mathrm{~h}$. In particular, in situ gels prepared from $1.2 \%$ alginate/0.4\% HPMC (G7) exhibited excellent analgesic (54.28 \%) and anti-inflammatory activity (51.6\% inhibition of rat paw edema) in the animal model of vertebral fracture.

Conclusion: The formulated in situ gels can potentially be used as implants for the treatment of patients with vertebral fracture.
\end{abstract}

Keywords: Ketorolac, Orthopaedic implant, Extended release, Analgesic, Anti-inflammation, Vertebral fracture

Tropical Journal of Pharmaceutical Research is indexed by Science Citation Index (SciSearch), Scopus, International Pharmaceutical Abstract, Chemical Abstracts, Embase, Index Copernicus, EBSCO, African Index Medicus, JournalSeek, Journal Citation Reports/Science Edition, Directory of Open Access Journals (DOAJ), African Journal Online, Bioline International, Open-J-Gate and Pharmacy Abstracts

\section{INTRODUCTION}

Vertebral fractures (VF) are common in patients suffering from osteoporosis and osteomyelitis and lead to severe pain at the fracture site. VF is a condition associated with acute back pain and may lead to chronic pain and loss of height. Upon stress induced by VF, the human body transmits alarming signals mediated by inflammation. Inflammation, in turn, is mediated through a number of chemical factors like bradykinin, histamine, prostaglandin and interleukin-1. First-line treatment of VF is associated with the administration of nonsteroidal anti-inflammatory drugs (NSAIDs) that reduce levels of the aforementioned chemical mediators, thereby relieving pain and inflammation [1-3]. However, complete VF 
treatment demands prolonged drug release in order to provide round-the-clock management of pain and inflammation.

As an example, ketorolac tromethamine $(\mathrm{KT})$ is a widely used NSAID for the management of moderate to severe pain associated with trauma, post-operative pain, breakthrough pain of cancer, VF as well as spinal injuries and is usually administered through oral, intramuscular or intravenous routes. $\mathrm{KT}$ acts by inhibiting cyclooxygenase-1 (COX-1) and COX-2 and has been reported to be 800 times more potent than aspirin [4-5]. Moreover, unlike narcotic analgesics, it is non-addictive and free of any respiratory side effects. However, since KT has a biological half-life $\left(t_{1 / 2}\right)$ of 4 to $6 \mathrm{~h}$, its frequent dosing is required in order to manage the chronic pain for a prolonged period.

Therefore, biodegradable, implantable drug delivery systems have been designed to release the drug of choice at the target site (for e.g., fractured vertebra) for an extended period of time. In this regard, various dosage forms or delivery systems including surgical implants and transdermal drug delivery systems have been investigated for prolonged drug release [6-8]. However, implantation of the aforementioned devices to a target site demands surgical intervention $[9,10]$, thereby reducing patient compliance. Recently, in situ gel systems have received considerable attention since they are in liquid state (before administration) and can transform into a stiff gel (viscous) format upon injection to the body cavity. In situ gel formation allows slow drug release, primarily by diffusion across the gel matrix. Additionally, in situ gels are advantageous in comparison to implantable drug delivery systems with respect to ease of their administration thereby leading to improved patient compliance [11].
Although a variety of biodegradable polymers have been employed for in situ gel formation, this study deals with the fabrication of alginate-based in situ gels for intrathecal delivery of KT. Alginates are naturally occurring anionic polysaccharides obtained from marine brown algae and consist of two monomeric units; $\beta$-Dmannuronic acid and $\alpha$-L-guluronic acid. Sodium salt of alginic acid (sodium alginate) shows gelation properties as a result of crosslinking of branched chain structure containing acidic contents with multivalent cations such as $\mathrm{Ca}^{2+}$, $\mathrm{Ba}^{2+}$, and $\mathrm{Al}^{3+}[12-15]$. Therefore, dilute aqueous solutions of alginate form firm gels upon addition of di- and trivalent ions (commonly present in simulated biological fluid (SBF)).

The present work deals with the development of alginate-based in situ gels for the delivery of KT. Intrathecal administration of these in situ gels could lead to reduced dosage frequency, prolonged drug release and ultimately improved patient compliance for the potential treatment of VF.

\section{EXPERIMENTAL}

\section{Materials}

Ketorolac tromethamine (KT), sodium alginate and hydroxypropylmethyl cellulose were purchased from Sigma Chemical (USA). All other chemicals were of analytical grade.

\section{Preparation of orthopaedic implants of KT}

On the basis of gelling capacity of various formulations as performed by hit and trial method, the composition of KT loaded in situ gel formulations were designed (Table 1).

Table 1: Composition of in situ gel formulations

\begin{tabular}{lcccccccc}
\hline \multirow{2}{*}{ Ingredient } & \multicolumn{9}{c}{ Concentration (w/v) } \\
\cline { 2 - 9 } KT & G1 & G2 & G3 & G4 & G5 & G6 & G7 & G8 \\
\cline { 2 - 9 } & 0.3 & 0.3 & 0.3 & 0.3 & 0.3 & 0.3 & 0.3 & 0.3 \\
Sodium alginate & 1.0 & 1.2 & 1.4 & 1.0 & 1.2 & 1.2 & 1.2 & 1.2 \\
HPMC & 0.2 & 0.2 & 0.2 & 1.0 & 0.1 & 0.3 & 0.4 & 0.5 \\
$\mathrm{NaCl}$ & 0.9 & 0.9 & 0.9 & 0.9 & 0.9 & 0.9 & 0.9 & 0.9 \\
$\begin{array}{l}\text { Sodium benzoate } \\
\begin{array}{l}\text { Phosphate buffer } \\
\text { pH 7.4,qs. to }\end{array}\end{array}$ & 0.01 & 0.01 & 0.01 & 0.01 & 0.01 & 0.01 & 0.01 & 0.01 \\
\hline $\begin{array}{l}\text { KT: Ketorolac tromethamine; HPMC: hydroxypropyl methylcellulose } \\
\text { KT: }\end{array}$ & 100 & 100 & 100 & 100 & 100 & 100 & 100 & 100 \\
\hline
\end{tabular}


Aqueous solutions of different HPMC concentrations were prepared by cold method and evaluated for gelling capacity and viscosity in order to identify the compositions suitable for in situ gel systems (Table 1). The alginate solutions were prepared by dispersing the required amount of sodium alginate in $75 \mathrm{~mL}$ distilled deionized water with continuous stirring until completely dissolved. Further, alginate/HPMC solutions were prepared by dispersing the required amount of HPMC in the desired concentration of sodium alginate with continuous stirring until completely dissolved. KT was dissolved in hydrochloric acid and the $\mathrm{pH}$ was adjusted to 6.7. Sodium benzoate was then added to the aforementioned solution. Finally, the drug solution was added to alginate or alginate/HPMC solution under constant stirring until a uniform, clear solution was obtained. Distilled deionized water was then added to make the volume up to $100 \mathrm{~mL}$. The gels were finally sterilized by ethylene oxide and stored in vacuum desiccator until further use.

\section{Physicochemical characterization}

The clarity of formulated solutions was determined by visual inspection under black and white background. Since $\mathrm{pH}$ is one of the most important factors involved in formulation design, $\mathrm{pH}$ of the prepared formulation was monitored using a $\mathrm{pH}$ meter.

\section{Drug content}

The drug content was determined by diluting $1 \mathrm{ml}$ of formulation with $10 \mathrm{ml}$ distilled water. This was further diluted with distilled water in the ratio 1 to 10 and the drug concentration was determined at $232 \mathrm{~nm}$ using UV-Vis spectrophotometer.

\section{Viscosity and rheology}

The viscosity of polymeric formulations either in solution or in gel made with artificial tear fluid, was determined using Brookfield viscometer DV II+Pro USA.

\section{In vitro release studies}

In vitro release of $\mathrm{KT}$ from formulations was studied using a fabricated $\mathrm{KC}$ cell testing apparatus. Briefly, a cellophane membrane was soaked overnight in freshly prepared dissolution medium (phosphate buffer, $\mathrm{pH}$ 7.4). The membrane was then inserted between the donor and receiver compartment of $\mathrm{KC}$ cell, following which $1 \mathrm{~mL}$ formulation was pipetted into this assembly. A $10 \mathrm{~mL}$ dissolution medium was maintained at $37 \pm 1{ }^{\circ} \mathrm{C}$ so that the membrane just touched the receptor medium surface. Dissolution medium was stirred with magnetic stirrer. Aliquots (each of $1 \mathrm{~mL}$ volume) were withdrawn at regular intervals and replaced by an equal volume of the receptor medium. The aliquots were then diluted with the receptor medium and analysed by UV spectrophotometer at $232 \mathrm{~nm}$.

\section{In vivo study}

Healthy male Wistar rats (200 - $250 \mathrm{~g})$ were used for the study. They were kept in cages under standard environmental conditions of light and temperature and were allowed free access to drinking water and standard diet. In vivo studies were carried out in compliance with Directive 2010/63/EU on the Handling of Animals Used for Scientific Purposes [16].

\section{In vivo analgesic activity}

Acute analgesic response by KT was assessed by acetic acid induced writhing method in rats. For this, rats were divided in three groups of six each. The first group served as the control group, wherein the rats received an appropriate volume of $2 \%$ Tween 80 only, p.o. To the second (test) group, KT loaded gel (G7) was injected intrathecally near the fractured vertebra (3.50 $\mathrm{mg} / \mathrm{kg}$ body mass). The third group was treated with standard ketorolac tablet $(0.33 \mathrm{mg} / \mathrm{kg}$ body mass) as slurry p.o. Three hours after treatment, the vertebra was fractured (around C2 and C4) by surgery. Total number of writhes, which was a parameter of chemically induced pain (i.e., constriction of abdomen, turning of trunk and extension of hind legs), was counted for a period of $20 \mathrm{~min}$. The analgesic effect was expressed as percentage reduction of writhes in comparison with the control group.

\section{In vivo anti-inflammatory study}

In the next set of experiments, in vivo antiinflammatory study was performed using the standard carrageenan induced hind rat paw edema model [14]. For this, rats were divided into control, standard and test groups of six animals each. Before treatment, initial paw volumes of all animals were measured using a mercury plethysmometer. Control group was given no drug, however, the rats received $2 \%$ xanthan gum orally in an appropriate volume. Standard group received free ketorolac (0.33 $\mathrm{mg} / \mathrm{kg}$ body mass, p.o.), while the test groups received KT loaded G7 gels $(3.50 \mathrm{mg} / \mathrm{kg}$ body mass), intrathecally. In order to induce paw edema, one hour following gel/drug administration, all rats were injected (subplantar 
route) with $0.1 \mathrm{~mL}$ of a $1 \% \mathrm{w} / \mathrm{v}$ homogeneous suspension of carrageenan (in double-distilled water) in the right paw. The hind paw volume was measured immediately $(0 \mathrm{~h})$ as well as at different time intervals, using a plethysmometer and expressed as percentage edema relative to the initial hind paw volume. The relative increase in paw volume was determined by measuring the paw volume after $3 \mathrm{~h}$ following the carrageenan administration. The percentage inhibition of edema, as an indicator of anti-inflammatory activity, was then compared with the control group.

\section{Statistical analysis}

The results in this study are expressed as mean \pm standard deviation (SD) and statistical analysis was performed by Student's t-test using Origin 1.5 (OriginLab Corporation, USA). A probability value of $p<0.05$ was considered to be significant.

\section{RESULTS}

In the first set of experiments, ion activated in situ gels were prepared using the cold method. Only those formulations that gelled instantaneously (<1 min) following their contact with SBF were selected for further study (Table $1)$.

Table 2: Physicochemical evaluation of KT loaded gels

\section{Physicochemical characterization of the gels}

Visual inspection revealed the formation of a translucent matrix following incubation of the formulations with SBF. The prepared gels were transparent at all $\mathrm{pH}$ values. Further, $\mathrm{pH}$ of all formulations was in acceptable range (Table 2) and the clarity of all formulations was satisfactory. Further, the formulations were slight yellow in colour.

The drug content was found to be in the acceptable range for all formulations. Percentage drug content in all formulations was in the range of 98.3 - $103.3 \%$ indicating uniform drug distribution in the studied formulations.

\section{Viscosity and rheological properties of KT loaded gels}

Three best formulations (G2, G4 and G7) were evaluated for viscosity using Brookfield viscometer DV II+Pro. The formulations exhibited pseudo-plastic rheology as evidenced by shear thinning and increase in shear rate as a function of angular velocity. The viscosity was however found to be dependent on polymeric content of the formulations and followed the order, G7>G2>G4.

\begin{tabular}{cccccc}
\hline$\bullet$ & Code & Appearance & Clarity & pH & $\begin{array}{c}\text { Drug } \\
\text { content (\%) }\end{array}$ \\
\hline$\bullet$ & G1 & Transparent & Clear & 6.4 & 100.20 \\
- G2 & G3 & Transparent & Clear & 6.7 & 102.12 \\
- & G4 & Transparent & Clear & 6.5 & 100.00 \\
- & G5 & Transparent & Clear & 6.7 & 103.10 \\
- & G6 & Transparent & Clear & 6.6 & 104.80 \\
- & G7 & Clear & 6.3 & 101.15 \\
- & G8 & Transparent & Clear & 6.7 & 99.10 \\
\hline
\end{tabular}

Table 2: Viscosity evaluation of KC loaded gels

\begin{tabular}{|c|c|c|c|c|c|c|}
\hline \multirow{3}{*}{$\begin{array}{c}\text { Variable } \\
\text { Angular } \\
\text { velocity } \\
\text { (rpm) }\end{array}$} & \multicolumn{6}{|c|}{ Viscosity of formulations (cps) } \\
\hline & \multicolumn{2}{|c|}{ G2 } & \multicolumn{2}{|c|}{ G4 } & \multicolumn{2}{|c|}{ G7 } \\
\hline & $\begin{array}{c}\text { Without } \\
\text { SBF }\end{array}$ & $\begin{array}{l}\text { With } \\
\text { SBF }\end{array}$ & $\begin{array}{c}\text { Without } \\
\text { SBF }\end{array}$ & $\begin{array}{l}\text { With } \\
\text { SBF }\end{array}$ & $\begin{array}{c}\text { Without } \\
\text { SBF }\end{array}$ & $\begin{array}{l}\text { With } \\
\text { SBF }\end{array}$ \\
\hline 10 & 7.20 & 22.9 & 5.06 & 18.09 & 10.5 & 24.9 \\
\hline 20 & 6.00 & 21.9 & 4.12 & 17.90 & 9.05 & 23.7 \\
\hline 30 & 5.00 & 20.5 & 3.00 & 16.50 & 8.12 & 22.7 \\
\hline 40 & 4.05 & 19.2 & 2.02 & 15.20 & 7.05 & 21.7 \\
\hline 50 & 3.00 & 18.0 & 1.11 & 14.00 & 6.00 & 20.6 \\
\hline
\end{tabular}


Table 4: In vivo analgesic and anti-inflammatory activity of KT loaded gel

\begin{tabular}{lccccc}
\hline Drug & $\begin{array}{c}\text { Oral dose }^{\mathrm{a}} \\
(\mathbf{m g} / \mathbf{k g})\end{array}$ & \multicolumn{2}{c}{ Analgesic Activity } & \multicolumn{2}{c}{ Anti-inflammatory activity } \\
\cline { 3 - 6 } & & No. of writhes & Analgesia (\%) & $\begin{array}{c}\text { Paw volume } \\
(\boldsymbol{m L})^{b, c}\end{array}$ & $\begin{array}{c}\text { Inhibition of } \\
\text { edema (\%) }\end{array}$ \\
\hline Control (2\% & - & $70 \pm 2$ & - & $0.95 \pm 0.04$ & - \\
Tween 80) & & & & & \\
KT (standard) & 0.33 & $26 \pm 2^{a}$ & 62.85 & $0.42 \pm 0.01^{a}$ & 55.8 \\
G7 & 3.4 & $32 \pm 1^{a}$ & 54.28 & $0.46 \pm 0.02^{a}$ & 51.6 \\
\hline
\end{tabular}

${ }^{\mathrm{a}}$ Dose equimolar to the parent drug calculated on the basis of drug contents; ${ }^{\mathrm{b}}$ Mean $\pm \mathrm{SEM}, \mathrm{n}=6$; $^{\mathrm{c}}$ Change in paw volume $3 \mathrm{~h}$ after carrageenan injection.; ${ }^{d} p<0.05$ vs. control

\section{In-vitro drug release}

In vitro drug release studies were carried out for all formulations using simulated biological fluid (SBF) as the dissolution medium. It was found that the percentage drug release was 88.67, $86.59,89.43,88.28,97.12,96.98,84.43$ and $91.38 \%$ for the formulation G1, G2, G3, G4, G5, G6, G7 and G8, respectively at the end of $12 \mathrm{~h}$. In order to gain insight into drug release mechanism from ion activated in situ gels, the release data was subjected to different modes of data treatment. The results indicated a zero order drug release profile from alginate based in situ gels, potentially as a result of diffusion from the gel matrix.

\section{In vivo analgesic and anti-inflammatory activity of KT loaded gels}

On the basis of physiochemical properties, rheological properties and in vitro release profile, formulation G7 was selected for determining the in vivo analgesic and anti-inflammatory potential of KT-loaded biodegradable implants on Wistar rats (Table 4).

Analgesic and anti- inflammatory activities of KT loaded G7 gels were found to be $54.28 \%$ and $51.6 \%$ respectively, and the effect was comparable to that of the free drug. The antiinflammatory activity was presented in terms of inhibition (\%) of paw edema using the standard carrageenan induced hind rat paw edema model. Taken together, the results of this study demonstrated the potential of KT loaded in situ gels (G7) in VF treatment due to high drug content, good rheological properties as well as analgesic and anti-inflammatory activity on Wistar rats.

\section{DISCUSSION}

Biodegradable polymers have shown potential as drug delivery systems in the form of implants and devices for bone repair. Apart from being nonmutagenic and non-cytotoxic, biodegradable polymers are metabolized in the body and eliminated by normal physiological pathways $[17,18]$. Of the various fabrication systems available, in situ gels show potential for local as well as systemic controlled drug delivery systems [19-21]. Further, since intrathecal KT (free drug) pre-treatments have been reported to reduce the spinal cord ischemic and VF injuries [22], this study employed in situ gels for intrathecal KT administration due to the disadvantages associated with the administration of free KT (e.g., poor drug stability, reduced half-life, frequent administration and hence reduced patient compliance).

Sodium alginate was used for the fabrication of ion activated in situ gels due to the property of its aqueous solutions to transform into stiff gels through ionic gelation in presence of divalent cations of SBF $\left(\mathrm{Ca}^{2+}\right)$. Further, alginate-based in situ gels have been reported to preserve their integrity without undergoing dissolution or erosion for prolonged period of time [23]. Moreover, the method used for fabrication of alginate-based in situ gels in the present study was simple and reproducible. Only those formulations were selected that demonstrated satisfactory attributes in terms of gelling capacity in the pre-formulation studies.

The initial drug release pattern from alginate based in situ gels was characteristic of hydrophilic matrices. This initial fast release of KT could be attributed to the fact that alginate gels were formulated in water and hence the polymer was completely hydrated. Incubation in SBF resulted in gelation and formation of a prehydrated matrix wherein hydration and water penetration no longer limited the drug release thereby leading to an apparent diffusion based controlled release. In particular, there was 11.19 $\%$ release of $\mathrm{KT}$ from formulation $\mathrm{G} 7$ after $0.5 \mathrm{~h}$, and $84.43 \%$ after $8 \mathrm{~h}$, and the drug release continued to rise thereafter.

Following comparison with other formulations, G7 (based on $1.2 \%$ alginate/0.4 \% HPMC) showed better ability to retain KT and hence was 
chosen for further studies. Additionally, the chosen formulation demonstrated analgesic as well as anti-inflammatory activity. Taken together, results of this study suggest that the alginate/HPMC aqueous system show potential as an in situ gel-forming system for intrathecal delivery in VF.

\section{CONCLUSION}

This work involved the development of alginatebased in situ gels for the delivery of KT. The gels demonstrated high drug encapsulation efficiency and can be employed for continuous and extended release of KT. The gels also exhibit analgesic and anti-inflammatory activity in vivo thereby indicating the potential for round-theclock management of pain and inflammation in patients with VF. However, further studies are required to determine the suitability of the formulation in patients with VF.

\section{ACKNOWLEDGEMENT}

Protocols for in vivo studies were approved by the Animal Care and Use Committee of The First Affiliated Hospital of Xinxiang Medical University, Weihui, China (approval reference no. 11/14/03/02).

\section{REFERENCES}

1. Block JH and Beale JM, Eds, Wilson and Giswolds Text book of Organic, Medicinal and Pharmaceutical Chemistry, 10th edn, Lippincott Raven Publishers, Philadelphia. 1998:711-713.

2. Foye WO. Principles of Medicinal Chemistry, 4th edn, B.I. Waverly Pvt Ltd, New Delhi. 1995: 535-540

3. Riggs BL, Melton LJ. The worldwide problem of osteoporosis: insights afforded by epidemiology, Bone 1995; 17(5): 505S-511S.

4. Litvak KM, McEvoy GK. Ketorolac, an injectable nonnarcotic analgesic. Clin. Pharm. 1990; 9: 921-935.

5. Buckley MMT, Brogden RN. Ketorolac: A review of its pharmacodynamics and pharmacokinetic properties, and therapeutic potential. Drugs 1990; 39: 86-109

6. Gratieri T, Pujol-Bello E, Gelfuso GM, Souza JG de, Lopez RF, Kalia YN. Iontophoretic transport kinetics of ketorolac in vitro and in vivo: demonstrating local enhanced topical drug delivery to muscle. Eur. J. Pharm. Biopharm. 2014; 86(2): 219-226.

7. Vyas S, Trivedi $P$, Chaturvedi SC, Ketorolac-dextran conjugates: Synthesis, in vitro and in vivo evaluation, Acta Pharm. 2007; 57L: 441-450.

8. Li C, Li C, LiU Z, Li Q, Yan X, LiU Y, Lu W. Enhancement in bioavailability of ketorolac tromethamine via intranasal in situ hydrogel based on poloxamer 407 and carrageenan. Int. J. Pharm. 2014; 474(1-2): 123-133.
9. Nitsch MJ, Banakar UV. Implant drug delivery. $J$ Biomater. Appl. 1994; 8: 247-284.

10. Iyer SS, Barr WH, Dance ME, Coleman PR, Karnes HT. $A$ 'biorelevant' system to investigate in vitro drug released from a naltrexone implant. Int. J. Pharm. 2007; 340: 104-118.

11. Shi Y, Li LC. Current advances in sustained-release systems for parenteral drug delivery. Expert Opin. Drug Deliv. 2005; 2(6): 1039-1058.

12. Sharma VK, Mazumdar B. Gastrointestinal transition and anti-diabetic effect of Isabgol husk microparticles containing gliclazide. Int. J. Biol. Macro. 2014; 66: 1525.

13. Soares JP, Santos JE, Chierice GO, Cavalheiro ETG. Thermal behavior of alginic acid and its sodium salt. Ecl. Quím., São. Paulo. 2004; 29(2): 53-56.

14. Zhang Xian-Zhao, Tian Fu-Jun, Hou Ya-Min, Ou ZhiHong. Preparation and in-vitro in-vivo characterization of polyelectrolyte alginate-chitosan complex based microspheres loaded with verapamil hydrochloride for improved oral drug delivery. J. Incl. Phenom. Macrocycl. Chem 2015; 81: 429-40.

15. Lanjhiyana SK, Bajpayee $P$, Kesavan K, Lanjhiyana $S$, Muthu MS. Chitosan-sodium alginate blended polyelectrolyte complexes as potential multiparticulate carrier system: colon-targeted delivery and gamma scintigraphic imaging. Expert Opin. Drug Deliv. 2013; 10: 5-15.

16. European Commission [homepage on the internet]. Directive 2010/63/EU on the protection of animals used for scientific purposes [cited 2015 March 01]. Available from: http://ec.europa.eu/environment/ chemicals/lab animals/legislation_en. $h$ tm

17. Van der Zee M., Biodegradability of polymers-Mechanism and evaluation methods. In Handbook of biodegradable polymers. Bastioli, C., Eds. Shropshire, Rapra Technology Limited: 2005; 23: 1-31.

18. Gao C, Gao J, You X, Huo S, Li X, Zhang Y, Zhang W. Fabrication Of Calcium Sulfate/PLGA Composite For Bone Repair. J Biomedical Material Res 2005; 73: 244253.

19. Yan S, Wang T, Feng L, Zhu J, Zhang K, Chen X, Cui L, Yin J. Injectable in situ self-cross-linking hydrogels based on poly (L-glutamic acid) and alginate for cartilage tissue engineering. Biomacromolecules. 2014; 15(12): 4495-508.

20. Zheng L, Jiang X, Chen X, Fan H, Zhang X. Evaluation of novel in situ synthesized nanohydroxyapatite/collagen/alginate hydrogels for osteochondral tissue engineering. Biomed. Mater. 2014; 9(6): 065004.

21. El Maghraby GM, Elzayat EM, Alanazi FK. Investigation of in situ gelling alginate formulations as a sustained release vehicle for co-precipitates of dextromethrophan and Eudragit S 100. Acta Pharm. 2014; 64(1): 29-44.

22. Hsieh YC, Liang WY, Tsai SK, Wong CS. Intrathecal ketorolac pretreatment reduced spinal cord ischemic injury in rats. Anesth. Analg. 2005; 100: 1134-1139. 
Cao et al

23. Semalty A, Pandey M, Adhikari L. Preparation and characterization of alginate microspheres and the effect of different types and concentration of crosslinking agents, Int. Res. J. Inventions Pharm. Sci. 2014; 2(1): 33-37. 